\title{
Sclerostin Antibody Improves Skeletal Parameters in a Brtl/+ Mouse Model of Osteogenesis Imperfecta
}

\author{
Benjamin P Sinder, ${ }^{1,2}$ Mary M Eddy, ${ }^{1}$ Michael S Ominsky, ${ }^{3}$ Michelle S Caird ${ }^{1}$ \\ Joan C Marini, ${ }^{4}$ and Kenneth M Kozloff ${ }^{1,2}$ \\ ${ }^{1}$ Orthopaedic Research Laboratories, Department of Orthopaedic Surgery, University of Michigan Ann Arbor, MI, USA \\ ${ }^{2}$ Department of Biomedical Engineering, University of Michigan, Ann Arbor, MI, USA \\ ${ }^{3}$ Department of Metabolic Disorders, Amgen, Inc., Thousand Oaks, CA, USA \\ ${ }^{4}$ Bone and Extracellular Matrix Branch, National Institute of Child Health and Human Development, NIH, Bethesda, MD, USA
}

\begin{abstract}
Osteogenesis imperfecta (OI) is a genetic bone dysplasia characterized by osteopenia and easy susceptibility to fracture. Symptoms are most prominent during childhood. Although antiresorptive bisphosphonates have been widely used to treat pediatric Ol, controlled trials show improved vertebral parameters but equivocal effects on long-bone fracture rates. New treatments for Ol are needed to increase bone mass throughout the skeleton. Sclerostin antibody ( $\mathrm{Scl}-\mathrm{Ab}$ ) therapy is potently anabolic in the skeleton by stimulating osteoblasts via the canonical wnt signaling pathway, and may be beneficial for treating OI. In this study, Scl-Ab therapy was investigated in mice heterozygous for a typical Ol-causing Gly $\rightarrow$ Cys substitution in col1a1. Two weeks of Scl-Ab successfully stimulated osteoblast bone formation in a knock-in model for moderately severe OI (Brtl/ +) and in WT mice, leading to improved bone mass and reduced long-bone fragility. Image-guided nanoindentation revealed no alteration in local tissue mineralization dynamics with $\mathrm{Scl}-\mathrm{Ab}$. These results contrast with previous findings of antiresorptive efficacy in Ol both in mechanism and potency of effects on fragility. In conclusion, short-term Scl-Ab was successfully anabolic in osteoblasts harboring a typical Ol-causing collagen mutation and represents a potential new therapy to improve bone mass and reduce fractures in pediatric OI. (C) 2013 American Society for Bone and Mineral Research.
\end{abstract}

KEY WORDS: OSTEOGENESIS IMPERFECTA; SCLEROSTIN ANTIBODY; COLLAGEN; BONE MASS; ANABOLIC THERAPY

\section{Introduction}

$\mathrm{O}$ steogenesis imperfecta (OI), or "brittle bone disease," is heritable disorder caused predominantly by mutations in type I collagen or in proteins that promote the folding or posttranslational modification of collagen. Patients with OI have increased bone fragility and are susceptible to fracture from minimal force, skeletal deformities, and growth deficiency. OI fragility symptoms are generally most prominent in children; the disorder ranges in severity from mild forms with slightly elevated fracture risk to perinatal lethality. ${ }^{(1)}$

Current treatment options for $\mathrm{Ol}$ focus on antiresorptive bisphosphonates (BPs), which have been shown effective at increasing vertebral areal bone mineral density (BMD) and height in clinical trials. However, BP effects in long bones are less evident, and most pediatric OI trials observe little or no functional benefit. ${ }^{(2-6)}$ Moreover, whereas BP therapy has been generally well tolerated in pediatric Ol patients, there are concerns about long-term retention of BPs in the skeleton and use in a growing population. ${ }^{(7)}$

Sclerostin antibody ( $\mathrm{SCl}-\mathrm{Ab})$ is a novel anabolic bone therapeutic presently in clinical trials for treatment of osteoporosis. ${ }^{(8)}$ Sclerostin is secreted primarily by osteocytes and negatively regulates bone formation by binding to the LRP4/5/6 complex, inhibiting anabolic canonical wnt signaling in osteoblasts. ${ }^{(9-11)}$ As a result, treatment with a neutralizing $\mathrm{Scl}-\mathrm{Ab}$ reduces sclerostin inhibition of canonical wnt signaling and is highly anabolic in preclinical models and in a phase 1 clinical trial of men and postmenopausal women. ${ }^{(8,12,13)}$ Whether Scl-Ab therapy is capable of stimulating osteoblast activity in cells harboring a typical Ol-causing mutation has yet to be demonstrated.

The Brtl/+ mouse is a knock-in model for moderately severe Type IV OI with a G349C point mutation on col1a1 that recreates an identical defect found in an Ol patient. ${ }^{(14)}$ The Brtl/+ mouse recapitulates multiple features of the observed clinical phenotype, including smaller size, reduced BMD, more severe

Received in original form February 28, 2012; revised form July 9, 2012; accepted July 16, 2012; accepted manuscript online July 26 , 2012. Address correspondence to: Kenneth M Kozloff, PhD, 2015 Biomedical Science Research Building, 109 Zina Pitcher Place, Ann Arbor, MI 48109-2200, USA E-mail: kenkoz@umich.edu

Journal of Bone and Mineral Research, Vol. 28, No. 1, January 2013, pp 73-80

DOI: 10.1002/jbmr.1717

(C) 2013 American Society for Bone and Mineral Research 
phenotype at young ages, increased bone brittleness, and increased bone turnover, ${ }^{(15,16)}$ making it an appropriate model for testing the anabolic efficacy of $\mathrm{Scl}-\mathrm{Ab}$ in Ol. The purpose of this study was to determine whether a short-term intervention with $\mathrm{Scl}-\mathrm{Ab}$ in the $\mathrm{Brtl} /+$ model of $\mathrm{Ol}$ would be effective at stimulating an anabolic skeletal response and improving long-bone strength.

\section{Materials and Methods}

Animals

Wild-type (WT) and Brtl/ + mice are maintained on a mixed background of Sv129/CD-1/C57BL/6S, and all Brtl/+ animals were the product of breeding male Brtl/ + mice with female WT mice. Eight-week-old male WT and Brtl/+ mice were randomly assigned to Scl-Ab (Scl-AbIV; Amgen, Thousand Oaks, CA, USA) treatment or vehicle (Veh) injection (PBS) with $n=7 /$ group. Sclerostin antibody was injected subcutaneously at $25 \mathrm{mg} / \mathrm{kg}$, two times per week, for 2 weeks. Calcein $(30 \mathrm{mg} / \mathrm{kg}$ ) was injected at the start of experiment, after 1 week, and 1 day prior to sacrifice by intraperitoneal injection to facilitate dynamic histomorphometry and nanoindentation placement. Body weights were recorded with each injection. Blood samples were collected at euthanasia by intracardiac puncture, and serum was separated by centrifuge and stored at $-80^{\circ} \mathrm{C}$ until analyzed by ELISA.

Left femurs were collected for micro-computed tomography $(\mu \mathrm{CT})$ and mechanical testing, and right femurs were collected for dynamic histomorphometry and nanoindentation tests. Both were stored at $-20^{\circ} \mathrm{C}$ in lactated Ringer's solution (LRS)-soaked gauze until testing or further specimen preparation. All protocols and procedures involving animals were approved by the University of Michigan's Committee on Use and Care of Animals.

\section{Serum markers}

To measure osteoblast activity, serum osteocalcin (OCN) was quantified with a commercially available ELISA kit (BT-470; BTI, Stoughton, MA, USA). To quantify osteoclast number, serum TRACP5b was measured with a commercially available solidphase immunofixed enzyme activity assay (MouseTRAP; IDS, Fountain Hills, AZ, USA). Both serum tests were performed in duplicate.

\section{$\mu \mathrm{CT}$}

Left femora were scanned in water using cone beam CT (eXplore Locus SP; GE Healthcare Pre-Clinical Imaging, London, ON, Canada). Scan parameters included a 0.5-degree increment angle, 4 frames averaged, an $80-k V p$ and $80-\mu \mathrm{A} \mathrm{X-ray} \mathrm{source} \mathrm{with}$ a $0.508-\mathrm{mm}$ Al filter to reduce beam hardening artifacts, and a beam flattener around the specimen holder. ${ }^{(17)}$ All images were reconstructed and calibrated at an $18-\mu \mathrm{m}$ isotropic voxel size to a manufacturer supplied phantom of air, water, and hydroxyapatite. Regions of interest (ROI) were located for both cortical and trabecular parameters. A diaphyseal cortical region of interest (ROI) spanning $15 \%$ of total femur length was located midway between the distal growth plate and third trochanter. Cortical bone was isolated with a fixed threshold of 2000 Hounsfield units (HU) for all experimental groups. Parameters including cortical thickness, cross sectional area, tissue mineral density (TMD), anterior-posterior bending moment of inertia, endosteal perimeter, and periosteal perimeter were quantified with commercially available software (MicroView v2.2 Advanced Bone Analysis Application; GE Healthcare Pre-Clinical Imaging, London, ON, Canada). A trabecular ROI 10\% of total femur length was located immediately proximal to the distal femoral growth plate and defined along the inner cortical surface with a splining algorithm. Due to the large differences in morphology induced by Scl-Ab treatment, a fixed threshold could not be utilized without bias. Trabecular metaphyseal bone was isolated with a more conservative autothresholding algorithm for each specimen based on the bimodal distribution between marrow and bone. ${ }^{(18)}$ Parameters including bone volume fraction (BV/TV), trabecular thickness (TbTh), and trabecular number (TbN) were quantified using standard stereology algorithms (MicroView v2.2). A 3D sphere-fitting algorithm was also used to confirm the stereology data for TbTh. ${ }^{(19)}$

\section{Mechanical testing (whole bone)-four-point bending}

Following $\mu \mathrm{CT}$ scanning, left femora were loaded to failure in four-point bending using a servohydraulic testing machine (MTS 858; MiniBionix, Eden Prairie, MN, USA). All specimens were kept hydrated in LRS-soaked gauze until mechanical testing. In the same mid-diaphyseal region analyzed by $\mu \mathrm{CT}$, the mid-diaphysis was loaded in four-point bending with the posterior surface oriented under tension. The distance between the wide, upper supports was $6.26 \mathrm{~mm}$, and the span between the narrow, lower supports $2.085 \mathrm{~mm}$. The vertical displacement rate of the fourpoint bending apparatus in the anterior-posterior direction was $0.5 \mathrm{~mm} / \mathrm{s}$. Force was recorded by a 50 -lb load cell (Sensotec, Columbus, $\mathrm{OH}, \mathrm{USA}$ ) and vertical displacement by an external linear variable differential transducer (LVDT; Lucas Schavitts, Hampton, VA, USA), both at $2000 \mathrm{~Hz}$. A custom MATLAB script was used to calculate stiffness, yield load, yield displacement, ultimate load, failure displacement, post-yield displacement, and energy to failure. Combining anterior-posterior bending moment of inertia data from $\mu \mathrm{CT}$ with mechanical stiffness from four-point bending, the estimated elastic modulus was calculated using standard beam theory as described. ${ }^{(15)}$

\section{Mechanical testing (tissue level)-fluorescent-guided nanoindentation}

Right femora were dehydrated, encased in epoxy (Kold Mount; Vernon-Benshoff, Albany, NY, USA), and cut transversely at the mid-diaphysis with a low-speed saw (IsoMet; Beuhler, Lake Bluff, IL, USA). The distal section of tissue was polished using progressive grades of silicon carbide abrasive paper (12002400-, and 4000-grit) under water irrigation for 2 minutes at each grade. To further decrease surface roughness, the encased specimens were polished on a felt pad for 5 minutes with a $0.25-\mu \mathrm{m}$ diamond suspension (Struers Inc., Cleveland, OH, USA). Specimens were then ultrasonically cleansed in a water bath for 10 minutes to remove surface debris, and glued to magnetic specimen plates for nanoindentation testing. The final root mean square (RMS) roughness of the specimens' surface was 
$10.0 \pm 3.7 \mathrm{~nm}$, as assessed with scanning probe microscopy of a $5-\mu \mathrm{m} \times 5-\mu \mathrm{m}$ region of the posterior mid-cortex.

A custom $950 \mathrm{TI}$ Tribolndenter (Hysitron, Minneapolis, MN, USA) instrumented with a fluorescent light-source and fluorescein isothiocyanate (FITC) filter allowed for simultaneous visualization of calcein labeling in specimens and accurate positioning of indents to locations matched for tissue age and treatment status with $0.5-\mu \mathrm{m}$ spatial resolution. Four ROIs were mechanically tested in the posterior aspect of the femoral cross section: the mid-cortex (defined as midway between the first calcein label on the periosteal and endosteal surfaces, if any); along the first calcein label on 15-day-old bone; along the second calcein label on 8-day-old bone; and along the third and outer calcein label along 1-day-old bone. Indentation consisted of loading a diamond Berkovich indenter tip (Hysitron, Minneapolis, MN, USA) into samples at $300 \mu \mathrm{N} / \mathrm{s}$, holding at a maximal load of $3000 \mu \mathrm{N}$ for $10 \mathrm{~s}$, and unloading at $300 \mu \mathrm{N} / \mathrm{s}$. The indentation modulus $\mathrm{E}$ was calculated from the load-displacement curves using the standard Oliver-Pharr method. ${ }^{(20)}$ Eight indents, $10 \mu \mathrm{m}$ apart, were made along each calcein label and the mid-cortex, with values averaged for each site and mouse.

\section{Dynamic histomorphometry}

Using the same specimens tested by nanoindentation, dynamic histomorphometry was performed at the mid-diaphysis on the first and third labels according to standard nomenclature. ${ }^{(21)}$ Briefly, fluorescent images were acquired using a Zeiss Axiovert 200M inverted microscope equipped with Apotome imaging system to minimize out-of-plane light, negating the need for thin sections. Fluorescent images were taken with a $\times 10$ objective of calcein (excitation 485/20 nm, emission: 540/25 nm) labels in bone. The $\times 10$ images of the cortex were merged into a single image (PhotoShop; Adobe, Inc.), and these merged images were analyzed using commercially available software (Bioquant Osteo v7.20.10; Bioquant, Nashville, TN, USA). Bone surface (BS), mineral apposition rate (MAR), mineralizing surface to bone surface (MS/BS), and bone formation rate (BFR) were quantified.

\section{Statistics}

Based on previous studies examining the phenotype of Brtl/+ at 8 weeks of age ${ }^{(15)}$ and on the therapeutic effects of BP in $\mathrm{Brtl} /{ }_{+}{ }^{(16)}$ a power analysis suggests that samples sizes of seven per genotype per group would sufficiently detect a therapeutic response equivalent to that of alendronate after 12 weeks of therapy. A multivariate ANOVA with least significant difference (LSD) post hoc was used to make all comparisons with the exception of nanoindentation data. For nanoindentation data along calcein labels, a repeated measures ANOVA was used with genotype and treatment status as between-subjects factors and tissue age as the within-subjects factor with simple contrasts to mid-cortical reference values. In all cases, $p<0.05$ was considered significant. All data is presented as mean \pm SD.

\section{Results}

Body weight and bone length remain unchanged with short-term Scl-Ab treatment

Consistent with the reduced body size with OI, body weight was significantly lower in male Brtl/ + $(25.4 \pm 1.8 \mathrm{~g})$ compared to male WT mice $(30.4 \pm 2.8 \mathrm{~g})$ at 8 weeks of age. Twice-weekly dosing of these mice with $25 \mathrm{mg} / \mathrm{kg} \mathrm{Scl-Ab}$ for 2 weeks did not significantly change body weight gains (data not shown). Scl-Ab treatment also had no effect on femoral length in WT mice $(15.9 \pm 0.6 \mathrm{~mm}$ Veh versus $16.0 \pm 0.5 \mathrm{~mm} \mathrm{Scl-Ab)}$ or the shorter Brtl/+ mice $(15.2 \pm 0.4 \mathrm{~mm}$ Veh versus $15.5 \pm 0.4 \mathrm{~mm} \mathrm{Scl-Ab})$ after 2 weeks.

\section{Serum markers show Brtl/+ anabolic response to $\mathrm{Scl}-\mathrm{Ab}$}

Untreated $\mathrm{Brtl} /+$ animals showed marginally elevated serum osteocalcin $(p=0.060)$ and serum TRACP5b $(p=0.083)$ relative to WT (Fig. 1), consistent with reported increases in Brtl/+ bone turnover. ${ }^{(16)} \mathrm{Scl}-\mathrm{Ab}$ therapy significantly increased serum osteocalcin in both WT and Brtl/+ (Fig. 1), demonstrating a rapid systemic anabolic response to therapy. Scl-Ab yielded trends toward reduced levels of serum TRACP5b in both WT $(p=0.10)$ and $\mathrm{Brtl} /+(p=0.082)$ mice, consistent with published

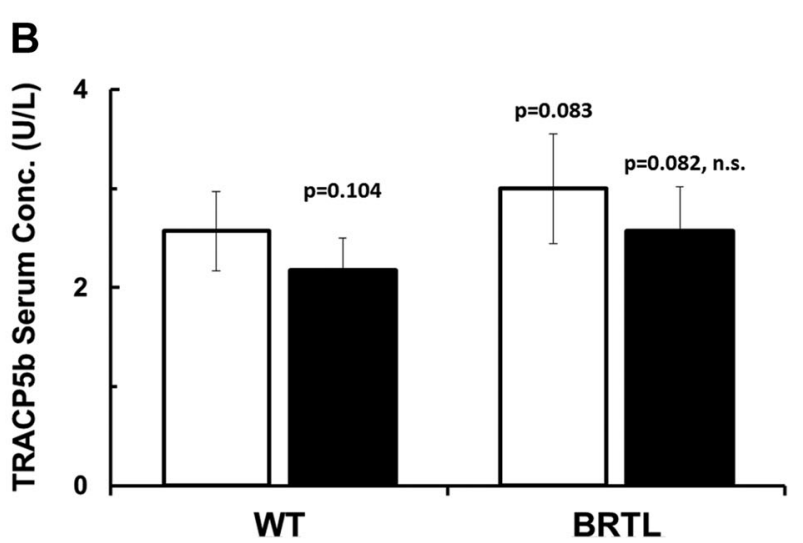

Fig. 1. Serum OCN $(A)$ is elevated and serum TRACP5b $(B)$ is reduced with Scl-Ab therapy in both WT and Brtl/+, suggesting a decoupled effect on bone formation and bone resorption. In vehicle-treated animals, Brtl/+ trends toward higher OCN levels in $A$ and TRACP5b levels in $B$, suggesting increased bone turnover in Brtl/+. ${ }^{*} p<0.05 \mathrm{Scl}-\mathrm{Ab}$ versus Veh, $+p<0.05 \mathrm{Brtl} /+\mathrm{Scl}-\mathrm{Ab}$ versus WT Veh. 
reports observing reduced serum markers of bone resorption in rats and humans. ${ }^{(8,12)}$

$\mathrm{Scl}-\mathrm{Ab}$ differentially increases cortical bone formation in $\mathrm{Brtl} /+$ and WT

At the femoral mid-shaft, $\mathrm{Scl}-\mathrm{Ab}$ treatment increased periosteal BFR/BS in Brtl/+ and WT animals by $76 \%$ and $108 \%$, respectively (Fig. 2). In Brtl/+, these increases were caused by a $50 \%$ increase in MS/BS, with no significant improvement in MAR. Conversely, WT improvements in BFR/BS resulted from a significant $76 \%$ increase in MAR with no significant change in MS/BS.

\section{$\mathrm{Scl}-\mathrm{Ab}$ increases trabecular bone apposition on existing} bone surfaces

$\mathrm{Brtl} /+$ animals have lower BV/TV relative to WT caused by reduced trabecular number, but not thickness (Fig. 3). Scl-Ab significantly increased distal femoral metaphyseal BV/TV in WT animals with a trend in $\mathrm{Brtl} /+(p=0.077)$. These improvements resulted from significant increases in trabecular thickness in both WT and Brtl/ +, with no change in trabecular number in either genotype.

Cortical shape and size, not mineralization, are increased with Scl-Ab

At the femoral mid-diaphysis, Brtl/+ animals have a reduced cortical thickness and area relative to WT as measured by $\mu \mathrm{CT}$ (Table 1). Scl-Ab treatment significantly increased both cortical thickness and area in Brtl/+ and WT, primarily through increased femoral cortical periosteal perimeter. Moreover, Scl-Ab treatment of Brtl/+ restored cortical thickness and area to levels not significantly different from WT Veh. Endocortical perimeter showed no significant differences with $\mathrm{Scl}-\mathrm{Ab}$ treatment in either WT or Brtl/+. In combination, these individual factors contributed to increasing trends in the anterior-posterior bending moment of inertia with Scl-Ab treatment in both WT and $\mathrm{Brtl} /+$, reflecting a structurally stronger bone. Mean cortical tissue mineralization (TMD) calculated by $\mu \mathrm{CT}$ was unaffected by $\mathrm{Scl}-\mathrm{Ab}$ in either WT or Brtl/+.

Sclerostin antibody improves whole-bone mechanical properties

$\mathrm{Brtl} /+$ femora have inherently lower ultimate load and stiffness ( $p=0.063$ ) compared to WT (Table 1$)$. Two weeks of Scl-Ab treatment significantly increased ultimate load and stiffness in both WT and Brtl/+ mice. Importantly, Scl-Ab treatment of Brtl/+ improved functional outcomes including ultimate load and stiffness to levels not significantly different from WT Veh, suggesting that the phenotypic deficit was rescued by therapy. The estimated tissue elastic modulus, as calculated by standard beam theory, was not affected by Scl-Ab treatment in WT or $\mathrm{Brtl} /+$, corroborating similar findings in TMD.

A hallmark feature of $\mathrm{Ol}$ bone is increased tissue brittleness. Postyield displacement (PYD) during four-point bending is a measure of ductility, an inverse indicator of material brittleness. Phenotypically, although Brtl/+ PYD was not significantly

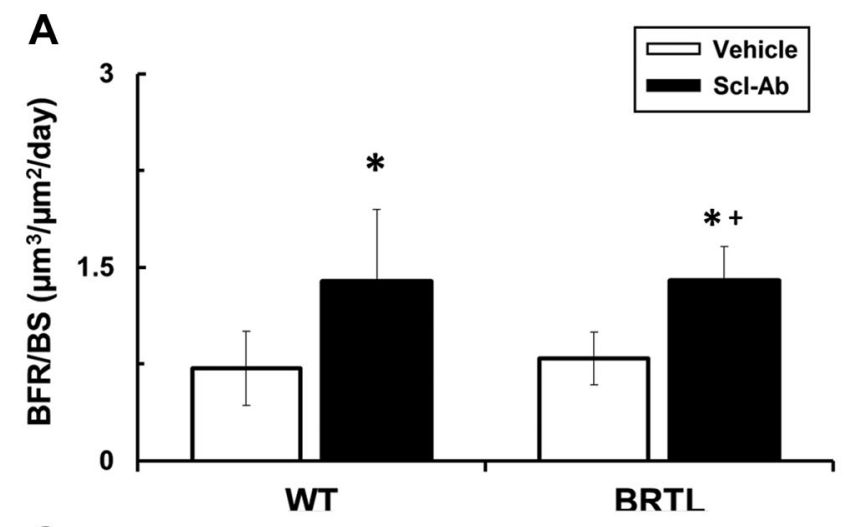

B
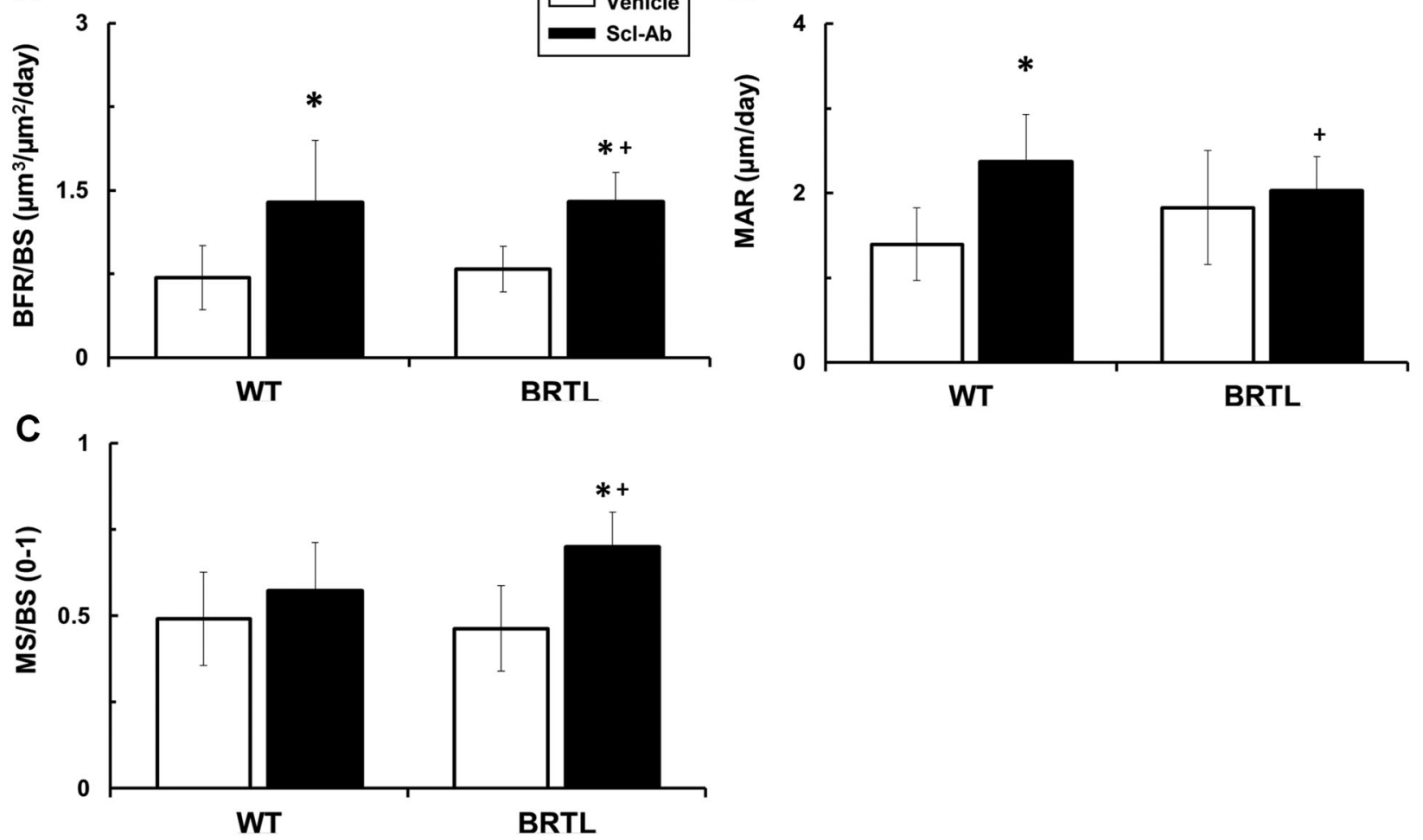

Fig. 2. Dynamic histomorphometry at the periosteal femoral mid-diaphysis reveals anabolic effect of Scl-Ab in WT and Brtl/+. Femoral cortical middiaphyseal periosteal BFR $(A)$ increases with Scl-Ab are a result of increased periosteal MAR $(B)$ in WT and periosteal MS/BS $(C)$ in Brtl/+. Brtl/ + Veh, $n=6$; all other groups $n=7 .{ }^{*} p<0.05 \mathrm{Scl}-\mathrm{Ab}$ versus Veh, $+p<0.05 \mathrm{Brtl} /+\mathrm{Scl}-\mathrm{Ab}$ versus WT Veh. 

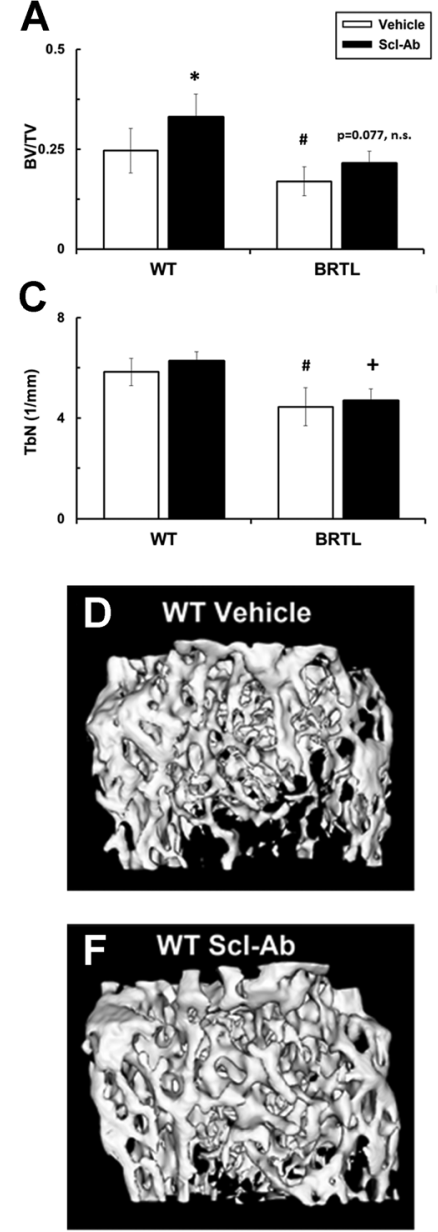

B

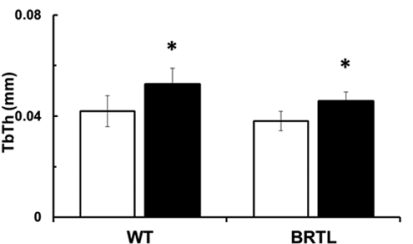

WT
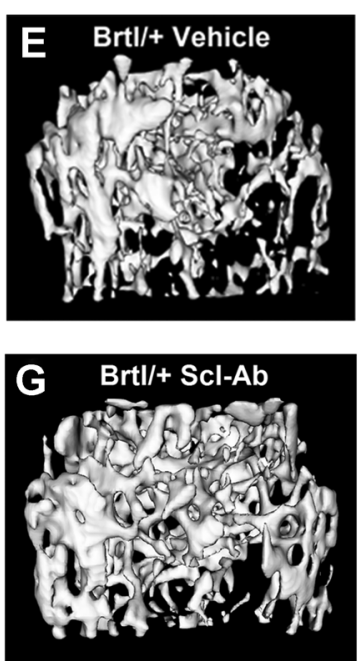

Fig. 3. $\mu C T$ of the distal femur metaphysis reveals anabolic changes with $\mathrm{Scl}-\mathrm{Ab}$ therapy. BV/TV $(A)$ and Tb.Th $(B)$ were improved by Scl-Ab with no effect on Tb.N (C). ( $D-G)$ Representative $\mu C T$ images of distal metaphyseal trabecular bone reflect these changes. For each experimental group, the animal with the median BV/TV value is shown. A conservative specimen specific threshold is applied as described in Materials and Methods. ${ }^{*} p<0.05 \mathrm{Scl}-\mathrm{Ab}$ versus Veh; \#p $<0.05 \mathrm{WT}$ Veh versus Brtl/+ Veh; $+p<0.05 \mathrm{Brtl} /+\mathrm{Scl}-\mathrm{Ab}$ versus WT Veh.

different than WT, five out of seven WT samples had a PYD greater than the largest Brtl/+ PYD value, consistent with previous reports of increased brittleness at a similar age. ${ }^{(15)}$ Unexpectedly, PYD was increased $324 \%$ with Scl-Ab in WT $(p<0.05)$ and $58 \%$ with $\mathrm{Scl}-\mathrm{Ab}$ in $\mathrm{Brtl} /+$, although failing to achieve statistical significance due to high variability. These data suggest $\mathrm{Scl}-\mathrm{Ab}$ reduced bone brittleness, improving bone ductility in both Brtl/+ and WT.

Nanoindentation reveals similar patterns of mineralization in Scl-Ab-treated animals

Nanoindentation measures local micromechanical properties associated with local tissue mineralization by measuring the tissue elastic modulus. Phenotypically, in the overall statistical model, genotype was a significant predictor, suggesting a greater tissue elastic modulus in Brtl/+ compared to WT. However, comparisons between genotypes at specific tissue ages were not significant. Nanoindentation revealed that $\mathrm{Scl}-\mathrm{Ab}$

treatment did not change the tissue elastic modulus of bone in either WT or Brtl/+. This indicates that despite the sudden change in osteoblastic anabolic function with Scl-Ab treatment, no differences in tissue elastic modulus were seen in bone grown under the influence of $\mathrm{Scl}$-Ab (Fig. 4). The tissue age at which newly formed bone achieved mature tissue elastic modulus was rapid, as only bone laid down 1 day prior to euthanasia had a significantly reduced elastic modulus relative to mid-cortical values consistent with the rapid establishment of mechanical properties which has been reported in rats. ${ }^{(22,23)}$ No difference was found between 2-week-old bone and 1-week-old bone relative to mid-cortical values.

\section{Discussion}

In this study, mice with a genetic knock-in that recreates a typical Ol-causing mutation in type I collagen showed a positive response following short-term $\mathrm{Scl}-\mathrm{Ab}$ therapy. Two weeks of Scl$\mathrm{Ab}$ significantly improved bone mass at both cortical and trabecular sites, and significantly reduced long bone fragility. In the context of existing anabolic and antiresorptive treatment options, $\mathrm{Scl}-\mathrm{Ab}$ is novel and has high potential as a therapeutic for Ol patients. Current anabolic treatment options have not shown uniform success when applied to OI. The anabolic bone agent teriparatide is currently approved as an osteoporosis therapy, but has been associated with osteosarcoma after longterm treatment in growing rats, ${ }^{(24)}$ and is therefore contraindicated for use in children with open growth plates. Growth hormone has also been implemented as an anabolic therapy for OI. Although effective in some patients at increasing BMD as well as improving bone histomorphometry, the benefits are selective for patients who respond to recombinant human growth hormone $(\mathrm{rGH})$ with increased linear growth. Other Ol patients treated with $\mathrm{rGH}$ experience no positive effect, and may exacerbate their existing low bone mass phenotype by increasing osteoclast surface. ${ }^{(25)}$

As a result of limited anabolic therapeutics, antiresorptive BPs have been widely used in OI. Several controlled pediatric OI clinical trials have demonstrated that BP treatment is effective at increasing vertebral BMD. ${ }^{(2-6)}$ Functionally, intravenous BPs are effective at increasing vertebral height, an indirect indicator of bone strength that suggests increased resistance to compression. ${ }^{(3,4)}$ However, in long bones a functional benefit is less clear, even in controlled trials of up to 125 patients. ${ }^{(1-6)}$

Although BP therapy increases areal BMD in pediatric OI populations, recent evidence suggests potential for several undesirable effects intrinsic to the BP mechanism of action. First, BP binds strongly to the skeleton and has been detected in urinary markers 8 years after cessation of treatment, resulting in long-term suppression of bone turnover. ${ }^{(26)}$ Long-term BP therapy has been associated with the accumulation of microdamage in several animal models. ${ }^{(27-31)} \mathrm{Brtl} /+$ mice have an increased propensity to form and accumulate microdamage ${ }^{(32)}$ and thus BP treatment may potentially exacerbate this effect. During growth, antiresorptive therapy results in retention of calcified cartilage near the growth plates. This retention of primary spongiosa manifests radiographically as sclerotic 
Table 1. Cortical $\mu \mathrm{CT}$ and Mechanical Properties

\begin{tabular}{|c|c|c|c|c|}
\hline & WT Veh & WT Scl-Ab & Brtl Veh & Brtl Scl-Ab \\
\hline \multicolumn{5}{|l|}{ Cortical $\mu \mathrm{CT}$} \\
\hline Thickness (mm) & $0.21 \pm 0.01$ & $0.24 \pm 0.01^{*}$ & $0.17 \pm 0.02^{\#}$ & $0.20 \pm 0.02^{*}$ \\
\hline Cross-sectional area $\left(\mathrm{mm}^{2}\right)$ & $1.01 \pm 0.10$ & $1.16 \pm 0.10^{*}$ & $0.76 \pm 0.10^{\#}$ & $0.91 \pm 0.10^{*}$ \\
\hline Endosteal perimeter (mm) & $4.51 \pm 0.36$ & $4.43 \pm 0.22$ & $4.09 \pm 0.13^{\#}$ & $4.21 \pm 0.18^{+}$ \\
\hline Periosteal perimeter (mm) & $5.80 \pm 0.42$ & $5.95 \pm 0.32$ & $5.15 \pm 0.16^{\#}$ & $5.48 \pm 0.21^{*}(p=0.06)$ \\
\hline Bending moment of inertia $\left(\mathrm{mm}^{4}\right)$ & $0.21 \pm 0.05$ & $0.23 \pm 0.04$ & $0.12 \pm 0.03^{\#}$ & $0.16 \pm 0.03(p=0.08, p=0.06)$ \\
\hline Tissue mineral density $\left(\mathrm{mg} / \mathrm{cm}^{3}\right)$ & $1052 \pm 43$ & $1051 \pm 50$ & $1081 \pm 39$ & $1092 \pm 41$ (n.s., $p=0.1$ ) \\
\hline \multicolumn{5}{|l|}{ Mechanical four-point bending } \\
\hline Ultimate load (N) & $27.1 \pm 4.5$ & $38.7 \pm 10.2^{*}$ & $18.2 \pm 5.0^{\#}$ & $26.8 \pm 7.8^{*}$ \\
\hline Stiffness (N/mm) & $237 \pm 28$ & $297 \pm 66^{*}$ & $192 \pm 35(p=0.063)$ & $256 \pm 33^{*}$ \\
\hline Energy to failure $(J)$ & $3.1 \pm 1.6$ & $10.6 \pm 7.6^{*}$ & $1.0 \pm 0.6$ & $2.0 \pm 1.1$ \\
\hline Post-yield displacement (mm) & $0.058 \pm 0.049$ & $0.247 \pm 0.143^{*}$ & $0.026 \pm 0.021$ & $0.041 \pm 0.037$ \\
\hline Estimated elastic modulus (GPa) & $4.6 \pm 1.2$ & $4.8 \pm 0.5$ & $6.0 \pm 1.2^{\#}$ & $6.0 \pm 0.7^{+}$ \\
\hline
\end{tabular}

Femoral mid-diaphyseal cortical microCT and mechanical four-point bending data.

$\mu \mathrm{CT}=$ micro-computed tomography; $\mathrm{WT}=$ wild-type; $\mathrm{Veh}=$ vehicle; Scl-Ab = sclerostin antibody.

${ }^{*} p<0.05 \mathrm{Scl}-\mathrm{Ab}$ versus Veh.

$\# p<0.05$ WT Veh versus Brtl/+ SclAb.

${ }^{+} p<0.05 \mathrm{Brtl} /+\mathrm{SclAb}$ versus WT Veh.

metaphyseal banding, coinciding with each BP treatment cycle, and these changes can bias measured BMD gains ${ }^{(33)}$ without concomitant improvements in strength. Previous studies of BP treatment in Brtl/+ found increased trabecular number, but not
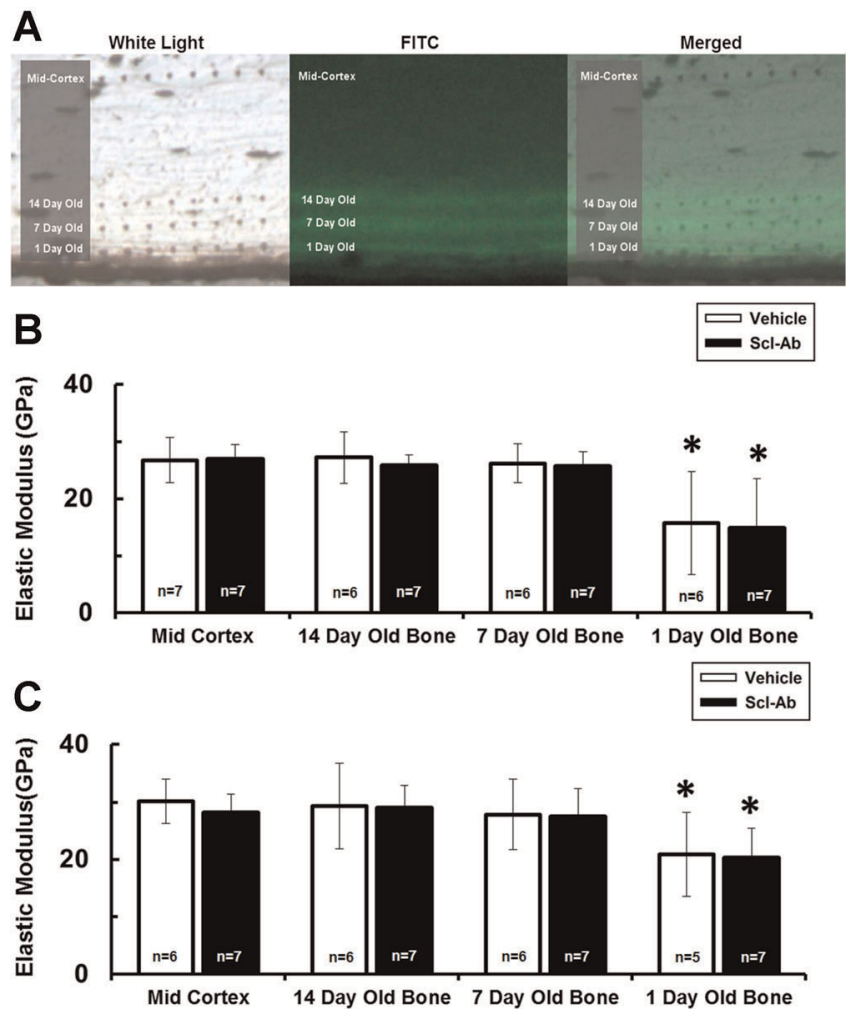

Fig. 4. Nanoindentation was performed along calcein labels to match tissue age between treated and untreated animals. (A) White light, FITC, and merged images showing placement of indents. Nanoindentation revealed no difference in tissue elastic modulus across treatment groups in WT $(B)$ and Brtl/ $+(C)$. *Significance relative to mid-cortical reference and denotes $p<0.05$. thickness. ${ }^{(33)}$ Notably, in the present study with $\mathrm{Scl}-\mathrm{Ab}$, gains in trabecular bone mass resulted from increased trabecular thickness, not number.

In this study, we found that unlike WT osteoblasts, Brtl/+ osteoblasts did not increase their MAR with Scl-Ab treatment. This lack of an MAR increase in Brtl/+ may be suggestive of defective osteoblasts unable to keep pace with $\mathrm{Scl}$-Ab-induced bone formation demands. In support of this, Brtl/+ mice demonstrate a mild delay in secretion of collagen in cell culture, and an enlarged endoplasmic reticulum (ER) has been observed in Brtl/+ fibroblasts, suggesting ER-stress correlated with collagen production, and possibly degradation of mutant collagen. ${ }^{(34)}$ As a consequence, Brtl/+ may respond to shortterm Scl-Ab by increasing mineralizing surface, and thus osteoblast recruitment, rather than individual cellular activity. This increase in mineralizing surface still leads to a significant increase in overall BFR, resulting in the improved bone size and strength observed. Whether this result also exists in the various Ol mouse models with different mutations will be important to contrast with the data presented here. Alternatively, animal age and treatment duration of the Brtl/+ mouse model used in this study may also modulate any Scl-Ab-induced MAR effect. Preliminary data from Brtl/+ mice treated for 5 weeks with Scl-Ab suggest a similar increase in MAR in both WT and Brtl/+ animals. ${ }^{(35)}$ Whereas a significant increase in periosteal perimeter was found in Brtl/+ when treated with sclerostin antibody, no significant differences were observed on the endosteal surface. This contrasts with previous findings of female rats treated with sclerostin antibody following ovariectomy, where a dominant treatment effect was observed on the endosteal bone surface. ${ }^{(12)}$ This may suggest a differential response of periosteal versus endosteal surface to $\mathrm{Scl}-\mathrm{Ab}$, dependent on animal age or hormonal status, reflecting either altered cell response to drug or differential bioavailability at these sites.

Consistent with previous studies of linear growth with $\mathrm{Scl}-\mathrm{Ab}$ or in male SOST knockout mice, ${ }^{(36,37)}$ we found no differences in 
either femur length or body mass, suggesting that longitudinal growth modulation is less sensitive than periosteal growth after 2 weeks of therapy. Unexpectedly, we found that Scl-Ab significantly reduced bone brittleness (increased PYD) in WT animals as measured by whole-bone mechanical four-point bending. Although reductions in bone brittleness were not statistically significant in Brtl/+ animals in this study, our finding of reduced brittleness in WT has broader implications about bone quality changes associated with $\mathrm{Scl}-\mathrm{Ab}$ treatment. Although the differing bone geometries induced by $\mathrm{Scl}-\mathrm{Ab}$ treatment raises the potential for experimental bias, the large magnitude of our observed difference in WT $324 \%$ increase in PYD) remains suggestive. In contrast to the $\mathrm{Scl}-\mathrm{Ab}$ data presented here, previous BP treatment in Brtl/+ found neither significant changes nor increasing trends in PYD. Moreover, BPtreated WT showed marginal reductions in PYD, reflecting no improvements in bone brittleness with BPs despite large gains in femoral bone mass. ${ }^{(33)}$ Interestingly, in the contralateral femurs of the same animals which demonstrated reductions in PYD, we did not observe any difference in the elastic modulus of bone formed under the influence of Scl-Ab by fluorescence-guided nanoindentation. These findings represent the first tissue-level mechanical test of bone formed during Scl-Ab treatment through coupling of fluorescent imaging to nanoindentation. Further studies are required to determine postyield behavior of bone at the tissue level, unlike those reported in this study.

The primary goals of this study were to demonstrate the potential for Scl-Ab efficacy at inducing an osteoblast response in $\mathrm{Brtl} /+$ at an age that has been well characterized (8 weeks). ${ }^{(15,16)}$ Although clinical efficacy will likely require an earlier onset of therapy and longer duration, these findings of significantly improved cortical bone mass, stiffness, and breaking loads with such a short duration of therapy are encouraging, particularly given the mixed response of BP efficacy at cortical sites. $^{(2-6)}$ Thus, these findings support further exploration of $\mathrm{Scl}-\mathrm{Ab}$ at earlier ages and for longer duration in treatment of $\mathrm{Ol}$.

In this study, Scl-Ab rapidly increased bone formation, resulting in increased cortical and cancellous bone volume and cortical bone strength without altering bone mineralization kinetics. Whereas Brtl/+ and WT both responded favorably to $\mathrm{Scl}-\mathrm{Ab}$, it is unknown if there are differences in sclerostin expression in Ol patients or OI mouse models throughout growth that could modulate the therapeutic effect. More broadly, as sclerostin mediates the Wnt signaling pathway, any phenotypic differences existing in Brtl/ +, including the receptors LRP4/5/6, competing ligands including DKK1, or regulation of b-catenin signaling may determine the skeletal $\mathrm{Scl}-\mathrm{Ab}$ response.

Furthermore, it should be recognized that some Scl-Ab studies have found a decrease in bone resorption outcomes. ${ }^{(8,12)}$ Given that an upregulation in bone resorption is phenotypic of Ol along with the trends of reduced serum TRACP5b data with Scl-Ab from this study, the osteoclast response will be explored in greater detail in future studies with longer-term therapy. Nevertheless, the trabecular $\mu C T$ data in this study shows a large increase in trabecular thickness with no change in trabecular number and is suggestive of a dominantly osteoblast-mediated effect.

In summary, we have demonstrated that 2 weeks of Scl-Ab therapy was capable of increasing bone formation rate in cells harboring a classical Ol-causing Gly $\rightarrow$ Cys substitution in col1a1. These gains lead to improved bone mass and whole-bone mechanical properties without altering the timing of tissue mineralization. Although BP therapy is in widespread use for pediatric Ol patients, there is pressing need for more effective anabolic therapeutics to improve patient outcomes. Scl-Ab is a unique and promising anabolic therapy that may be particularly useful for the treatment of pediatric OI. The present data suggest $\mathrm{Scl}-\mathrm{Ab}$ may be beneficial for the treatment of Ol patients by stimulating the osteoblasts and reducing fracture risk.

\section{Disclosures}

MSO is an employee and stock owner of Amgen, Inc. All other authors state that they have no conflicts of interest.

\section{Acknowledgments}

We thank Bonnie Nolan and Logan White for their contributions. $\mathrm{Scl}-\mathrm{Ab}$ was provided by Amgen and UCB Pharma.

Authors' roles: Study designed and conducted by BPS, MME, and KMK. Data collected by BPS and MME. Data analyzed and interpreted by BPS, MSO, MSC, JCM, and KMK. Manuscript written and approved by all authors. KMK takes responsibility for the integrity of the data analysis.

\section{References}

1. Forlino A, Cabral WA, Barnes AM, Marini JC. New perspectives on osteogenesis imperfecta. Nat Rev Endocrinol. 2011;7:540-57.

2. Sakkers R, Kok D, Engelbert R, van Dongen A, Jansen M, Pruijs $H$, Verbout A, Schweitzer D, Uiterwaal C. Skeletal effects and functional outcome with olpadronate in children with osteogenesis imperfecta: a 2-year randomised placebo-controlled study. Lancet. 2004;363: 1427-31.

3. Gatti D, Antoniazzi F, Prizzi R, Braga V, Rossini M, Tatò L, Viapiana O, Adami S. Intravenous neridronate in children with osteogenesis imperfecta: a randomized controlled study. J Bone Miner Res. 2005;20:758-63.

4. Letocha AD, Cintas HL, Troendle JF, Reynolds JC, Cann CE, Chernoff EJ, Hill SC, Gerber LH, Marini JC. Controlled trial of pamidronate in children with types III and IV osteogenesis imperfecta confirms vertebral gains but not short-term functional improvement. J Bone Miner Res. 2005;20:977-86.

5. Rauch F, Munns CF, Land C, Cheung M, Glorieux FH. Risedronate in the treatment of mild pediatric osteogenesis imperfecta: a randomized placebo-controlled study. J Bone Miner Res. 2009;24:1282-9.

6. Ward LM, Rauch F, Whyte MP, D'Astous J, Gates PE, Grogan D, Lester EL, McCall RE, Pressly TA, Sanders JO, Smith PA, Steiner RD, Sullivan E, Tyerman G, Smith-Wright DL, Verbruggen N, Heyden N, Lombardi A, Glorieux $\mathrm{FH}$. Alendronate for the treatment of pediatric osteogenesis imperfecta: a randomized placebo-controlled study. J Clin Endocrinol Metab. 2011;96:355-64.

7. Whyte MP, McAlister WH, Novack DV, Clements KL, Schoenecker PL, Wenkert D. Bisphosphonate-induced osteopetrosis: novel bone modeling defects, metaphyseal osteopenia, and osteosclerosis fractures after drug exposure ceases. J Bone Miner Res. 2008;23: 1698-707.

8. Padhi D, Jang G, Stouch B, Fang L, Posvar E. Single-dose, placebocontrolled, randomized study of AMG 785, a sclerostin monoclonal antibody. J Bone Miner Res. 2011;26:19-26. 
9. Poole KE, van Bezooijen RL, Loveridge N, Hamersma H, Papapoulos SE, Löwik CW, Reeve J. Sclerostin is a delayed secreted product of osteocytes that inhibits bone formation. FASEB.J. 2005;19:1842-4.

10. Li X, Zhang Y, Kang H, Liu W, Liu P, Zhang J, Harris SE. Wu D. Sclerostin binds to LRP5/6 and antagonizes canonical Wnt signaling. J Biol Chem. 2005;280:19883-7.

11. Leupin O, Piters E, Halleux C, Hu S, Kramer I, Morvan F, Bouwmeester T, Schirle M, Bueno-Lozano M, Fuentes FJ, Itin PH, Boudin E, de Freitas F, Jennes K, Brannetti B, Charara N, Ebersbach H, Geisse S, Lu CX, Bauer A, Van Hul W, Kneissel M. Bone overgrowth-associated mutations in the LRP4 gene impair sclerostin facilitator function. J Biol Chem. 2011;286:19489-500.

12. Li X, Ominsky MS, Warmington KS, Morony S, Gong J, Cao J, Gao Y, Shalhoub V, Tipton B, Haldankar R, Chen Q, Winters A, Boone T, Geng Z, Niu QT, Ke HZ, Kostenuik PJ, Simonet WS, Lacey DL, Paszty C. Sclerostin antibody treatment increases bone formation, bone mass, and bone strength in a rat model of postmenopausal osteoporosis. J Bone Miner Res. 2009;24:578-88.

13. Ominsky MS, Vlasseros F, Jolette J, Smith SY, Stouch B, Doellgast G, Gong J, Gao Y, Cao J, Graham K, Tipton B, Cai J, Deshpande R, Zhou L, Hale MD, Lightwood DJ, Henry AJ, Popplewell AG, Moore AR, Robinson MK, Lacey DL, Simonet WS, Paszty C. Two doses of sclerostin antibody in cynomolgus monkeys increases bone formation, bone mineral density, and bone strength. J Bone Miner Res. 2010;25: 948-59.

14. Forlino A, Porter FD, Lee EJ, Westphal H, Marini JC. Use of the Cre/lox recombination system to develop a non-lethal knock-in murine model for osteogenesis imperfecta with an $\alpha 1$ (I) G349C substitution. J Biol Chem. 1999;274:37923-31.

15. Kozloff KM, Carden A, Bergwitz C, Forlino A, Uveges TE, Morris MD, Marini JC, Goldstein SA. Brittle IV mouse model for osteogenesis imperfecta IV demonstrates postpubertal adaptations to improve whole bone strength. J Bone Miner Res. 2004;19:614-22.

16. Uveges TE, Collin-Osdoby P, Cabral WA, Ledgard F, Goldberg L, Bergwitz C, Forlino A, Osdoby P, Gronowicz GA, Marini JC. Cellular mechanism of decreased bone in Brtl mouse model of Ol: imbalance of decreased osteoblast function and increased osteoclasts and their precursors. J Bone Miner Res. 2008;23:1983-94.

17. Meganck JA, Kozloff KM, Thornton MM, Broski SM, Goldstein SA. Beam hardening artifacts in micro-computed tomography scanning can be reduced by X-ray beam filtration and the resulting images can be used to accurately measure BMD. Bone. 2009;45:1104-16.

18. Otsu N. A threshold selection method from gray-level histograms. IEEE Trans Syst Man Cybern. 1979;9:62-6.

19. Hildebrand T, Rüegsegger P. A new method for the model-independent assessment of thickness in three-dimensional images. J Microsc. 1997; 185:67-75.

20. Oliver WC, Pharr GM. An improved technique for determining hardness and elastic modulus using load and displacement sensing indentation experiments. J Mater Res. 1992;7:1565.

21. Parfitt AM, Drezner MK, Glorieux FH, Kanis JA, Malluche H, Meunier PJ, Ott SM, Recker RR. Bone histomorphometry: standardization of nomenclature, symbols, and units. Report of the ASBMR Histomorphometry Nomenclature Committee. J Bone Miner Res. 1987; 2:595-5610.

22. Busa B, Miller LM, Rubin CT, Qin Y-X, Judex S. Rapid establishment of chemical and mechanical properties during lamellar bone formation. Calcif Tissue Int. 2005;77:386-94.

23. Donnelly E, Boskey AL, Baker SP. van der Meulen MCH. Effects of tissue age on bone tissue material composition and nanomechanical properties in the rat cortex. J Biomed Mater Res A. 2010;92: 1048-56.

24. Vahle JL, Sato M, Long GG, Young JK, Francis PC, Engelhardt JA, Westmore MS, Linda Y, Nold JB. Skeletal changes in rats given daily subcutaneous injections of recombinant human parathyroid hormone (1-34) for 2 years and relevance to human safety. Toxicol Pathol. 2002;30:312-21.

25. Marini JC, Hopkins E, Glorieux FH, Chrousos GP, Reynolds JC, Gundberg CM, Reing CM. Positive linear growth and bone responses to growth hormone treatment in children with types III and IV osteogenesis imperfecta: high predictive value of the carboxyterminal propeptide of type I procollagen. J Bone Miner Res. 2003;18:237-43.

26. Papapoulos SE, Cremers SCLM. Prolonged bisphosphonate release after treatment in children. N Engl J Med. 2007;356:1075-6.

27. Komatsubara S, Mori S, Mashiba T, Li J, Nonaka K, Kaji Y, Akiyama T, Miyamoto K, Cao Y, Kawanishi J, Norimatsu H. Suppressed bone turnover by long-term bisphosphonate treatment accumulates microdamage but maintains intrinsic material properties in cortical bone of dog rib. J Bone Miner Res. 2004 Jun; 19(6):999-1005.

28. Li J, Mashiba T, Burr DB. Bisphosphonate treatment suppresses not only stochastic remodeling but also the targeted repair of microdamage. Calcif Tissue Int. 2001;69:281-6.

29. Mashiba T, Hirano T, Turner CH, Forwood MR, Johnston CC, Burr DB. Suppressed bone turnover by bisphosphonates increases microdamage accumulation and reduces some biomechanical properties in dog rib. J Bone Miner Res. 2000;15:613-20.

30. Mashiba T, Turner CH, Hirano T, Forwood MR, Johnston CC, Burr DB. Effects of suppressed bone turnover by bisphosphonates on microdamage accumulation and biomechanical properties in clinically relevant skeletal sites in beagles. Bone. 2001;28:524-31.

31. Allen MR, Reinwald S, Burr DB. Alendronate reduces bone toughness of ribs without significantly increasing microdamage accumulation in dogs following 3 years of daily treatment. Calcif Tissue Int. 2008;82: 354-60.

32. Davis MS, Kovacic BL, Marini JC, Shih AJ, Kozloff KM. Increased susceptibility to microdamage in Brtl/+ mouse model for osteogenesis imperfecta. Bone. 2012;50:784-91.

33. Uveges TE, Kozloff KM, Ty JM, Ledgard F, Raggio CL, Gronowicz G, Goldstein SA, Marini JC. Alendronate treatment of the Brtl osteogenesis imperfecta mouse improves femoral geometry and load response before fracture but decreases predicted material properties and has detrimental effects on osteoblasts and bone formation. J Bone Miner Res. 2009;24:849-59.

34. Forlino A, Kuznetsova NV, Marini JC, Leikin S. Selective retention and degradation of molecules with a single mutant [alpha]1(I) chain in the Brtl IV mouse model of OI. Matrix Biol. 2007;26:604-14.

35. Reich A, Cabral W, Marini J. Altered transcript pattern during osteoblast differentiation associated with improved bone phenotype in homozygous osteogenesis imperfecta Brtl mice. J Bone Miner Res. 2011;26(Suppl 1).

36. Marenzana M, Greenslade K, Eddleston A, Okoye R, Marshall D, Moore A, Robinson MK. Sclerostin antibody treatment enhances bone strength but does not prevent growth retardation in young mice treated with dexamethasone. Arthritis Rheum. 2011;63:2385-95.

37. Li X, Ominsky MS, Niu QT, Sun N, Daugherty B, D'Agostin D, Kurahara C, Gao Y, Cao J, Gong J, Asuncion F, Barrero M, Warmington K, Dwyer D, Stolina M, Morony S, Sarosi I, Kostenuik PJ, Lacey DL, Simonet WS, $\mathrm{Ke} \mathrm{HZ}$, Paszty C. Targeted deletion of the sclerostin gene in mice results in increased bone formation and bone strength. J Bone Miner Res. 2008;23:860-9. 